\title{
Visual attention in children with migraine: the importance of prophylaxis
}

\author{
Thaís Rodrigues Villa, Andréa Regina Correa Moutran, Alberto Alain Gabbai, Deusvenir de Souza Carvalho \\ Division of Investigation and Treatment of Headaches (DITH), Department of Neurology and Neurosurgery \\ Federal University of São Paulo - Unifesp, São Paulo,SP, Brazil
}

Villa TR, Moutran AR, Gabbai AA, Carvalho DS. Visual attention in children with migraine: the importance of prophylaxis. Headache Medicine. 2012;3(4):215-7

\section{INTRODUCTION}

Migraine and probable migraine in childhood has prevalence rates that range between $3.76 \%$ and $17.1 \%$ in Brazil. (1) Children with migraine have attention deficits, and experience negative consequences with regard to school performance as well as familial and social relationships. ${ }^{(2-3)}$

A possible role for the neurotransmitters was suggested, as was a close link between migraine and visual attention deficits. ${ }^{(3)}$ In continuation of our line of research, ${ }^{(3)}$ we herein aimed to evaluate the visual attention performance of children undergoing migraine prophylaxis in comparison with children with untreated migraine and a healthy control group.

\section{METHODS}

Eighty-two children were enrolled and divided into three groups: untreated migraine $(n=30)$, migraine prophylaxis $(n=22)$, and control $(n=30)$. Participants in the untreated migraine group had been newly admitted to the outpatient childhood headache service at the Division of Investigation and Treatment of Headaches (DITH) of the Federal University of São Paulo; all were diagnosed with migraine with $(n=5)$ or without aura ( $n=25$ ) according to the International Headache Society criteria (ICDH-II, 2004).(4) They were aged 8-12 years (mean age, $10.8 \pm 1.5$ years); 15 were boys. On average, they experienced $5.8 \pm 3.2$ days of headache per month, confirmed by a 30-day headache diary, without previous or current use of migraine prophylaxis. However, all children were free of pain and migraine symptoms in the two days preceding the assessment.

Participants in the migraine prophylaxis group were regular patients at DITH; all were diagnosed with migraine with $(n=6)$ or without aura $(n=16)$. They were aged 8-12 years (mean age, $10.8 \pm 1.5$ years); 10 were boys. They had experienced an average of $6.3 \pm 2.0$ days of headache per month prior to treatment. Prophylactic treatment started 3-6 months before evaluation, and children were free of pain and migraine symptoms in the two months preceding the assessment. Eight of them were taking sodium valproate (500-1000 $\mathrm{mg} /$ day), six of them propranolol (40-80 mg/day), five amitriptyline (25-50 mg/day), and three flunarizine (5 mg$10 \mathrm{mg} /$ day). All of these prophylactic drugs have been shown to be well tolerated and efficient in the treatment of migraine in this age group. The control group comprised children without headache aged $9.9 \pm 1.3$ years; 16 were boys. Children were selected through questionnaires completed by parents in two public schools in the city of São Paulo.

All children were subjected to medical and psychological evaluations. They were also evaluated using the Weschler Intelligence Scale for Children, $3^{\text {rd }}$ edition (WISC-III) which was applied by a DITH psychologist. Exclusion criteria were as follows: any other concomitant systemic illness; abnormalities revealed on neurological examination; intelligence quotient (IQ) less than 80; psychiatric disturbances; learning disabilities; history of epilepsy; head trauma or use of other drugs acting on the central nervous system, including alcohol consumption, smoking, and illicit drugs abuse; prior use of migraine prophylaxis (in the untreated migraine group) and a history of episodes of primary headache (in the control group).

Each child was subjected to the following visual attention tests, between July 2010 and January 2012 under the direction of a DITH psychologist: Trail Making Tests (TMT) parts A and B), the Letter - Cancellation Test and the Test of Visual Attention $3^{\text {rd }}$ edition, a computerized test, standardized for the Brazilian population. 
All children were students of public schools in São Paulo city and had similar socio economic backgrounds. None of them had a prior history of frequent school absences. Informed consent was obtained from the parents, and the study was approved by our Local Research Ethics Committee. For statistical analysis, the Student t-test and Mann-Whitney test were used. The significance level was set at $p<0.05$.

\section{RESULTS}

Children with untreated migraine performed significantly worse in TMT parts $A$ and $B(p<0.001)$ and had a greater number of action errors in tasks $1(p=0.032)$ and 2 ( $p=0.015)$ of the Visual Attention Test when compared with control group (Table 1). Children with

\begin{tabular}{|c|c|c|}
\hline Visual attention tests & Migraine group & Control group \\
\hline Trail making test $A$ & $23,0 \pm 12,6$ & $17,5 \pm 4,5(p=0,030)$ \\
\hline Trail making test $B$ & $66,6 \pm 36,6$ & $41,7 \pm 13,8(p=0,001)$ \\
\hline Letter cancellation test & $148,4 \pm 55,1$ & $129,4 \pm 32,5(p=0,111)$ \\
\hline $\begin{array}{l}\text { Cancellation omission } \\
\text { errors }\end{array}$ & $3,3 \pm 3,7$ & $2,9 \pm 3,3(p=0,851)$ \\
\hline Reaction time task 1 & $0,5 \pm 0,1$ & $0,6 \pm 0,1(p=0,073)$ \\
\hline Reaction time task 2 & $0,6 \pm 0,1$ & $0,6 \pm 0,1(p=0,799)$ \\
\hline Reaction time task 3 & $0,5 \pm 0,1$ & $0,5 \pm 0,2(p=0,960)$ \\
\hline Omission errors tark 1 & $2,2 \pm 2,8$ & $1,0 \pm 1,3(=0,108)$ \\
\hline Omission errors task 2 & $2,8 \pm 2,7$ & $1,9 \pm 1,6(p=0,221)$ \\
\hline Omission errors task 3 & $0,2 \pm 0,4$ & $0,1 \pm 0,3(p=0,499)$ \\
\hline Action errors task 1 & $2,4 \pm 1,7$ & $1,6 \pm 1,3(p=0,032)$ \\
\hline Action errors task 2 & $5,5 \pm 3,5$ & $3,4 \pm 2,7(p=0,015)$ \\
\hline Action errors task 3 & $2,4 \pm 5,7$ & $0,6 \pm 1,0(p=0,138)$ \\
\hline
\end{tabular}

Table 2 - Results of visual attention assessment in Migraine and Migraine prophylaxis groups

\begin{tabular}{lrr}
\hline Visual attention tests & Migraine group & $\begin{array}{r}\text { Migraine prophylaxisl } \\
\text { group }\end{array}$ \\
\hline Trail making test A & $23,0 \pm 12,6$ & $17,1 \pm 8(p=0,061)$ \\
Trail making test B & $66,6 \pm 36,6$ & $43 \pm 19,6(p=0,004)$ \\
Letter cancellation test & $148,4 \pm 55,1$ & $121,5 \pm 39,7(p=0,057)$ \\
Cancellation omission & $3,3 \pm 3,7$ & $2,5 \pm 2,9(p=0,412)$ \\
errors & & \\
Reaction time task 1 & $0,5 \pm 0,1$ & $0,5 \pm 0,05(p=0,603)$ \\
Reaction time task 2 & $0,6 \pm 0,1$ & $0,6 \pm 0,1(p=0,495)$ \\
Reaction time task 3 & $0,5 \pm 0,1$ & $0,5 \pm 0,09(p=0,511)$ \\
Omission errors tark 1 & $2,2 \pm 2,8$ & $0,9 \pm 0,7(p=0,019)$ \\
Omission errors task 2 & $2,8 \pm 2,7$ & $2,3 \pm 1,8(p=0,446)$ \\
Omission errors task 3 & $0,2 \pm 0,4$ & $0,05 \pm 0,2(p=0,083)$ \\
Action errors task 1 & $2,4 \pm 1,7$ & $2,0 \pm 1,8(p=0,228)$ \\
Action errors task 2 & $5,5 \pm 3,5$ & $3,6 \pm 2,9(p=0,051)$ \\
Action errors task 3 & $5,5 \pm 3,5$ & $0,4 \pm 0,8(p=0,057)$ \\
\hline
\end{tabular}

Table 3 - Results of visual attention assessment in Migraine prophylaxis and Control groups

\begin{tabular}{lrr} 
Visual attention tests & $\begin{array}{c}\text { Migraine } \\
\text { prophylaxisl } \\
\text { group }\end{array}$ & Control group \\
\hline Trail making test A & $17,1 \pm 8$ & $17,5 \pm 4,5(p=0,853)$ \\
Trail making test B & $43 \pm 19,6$ & $41,7 \pm 13,8(p=0,775)$ \\
Letter cancellation test & $121,5 \pm 39,7$ & $129,4 \pm 32,5(p=0,431)$ \\
Cancellation omission & $2,5 \pm 2,9$ & $2,9 \pm 3,3(p=0,654)$ \\
errors & $0,5 \pm 0,05$ & $0,6 \pm 0,1(p=0,038)$ \\
Reaction time task 1 & $0,6 \pm 0,1$ & $0,6 \pm 0,1(p=0,654)$ \\
Reaction time task 2 & $0,6 \pm 0,1$ & $0,5 \pm 0,2(p=0,594)$ \\
Reaction time task 3 & $0,9 \pm 0,7$ & $1,0 \pm 1,3(p=0,638)$ \\
Omission errors tark 1 & $2,3 \pm 2,0$ & $0,9 \pm 0,7(p=0,428)$ \\
Omission errors task 2 & $0,05 \pm 0,2$ & $0,1 \pm 0,3(p=0,264)$ \\
Omission errors task 3 & $2,0 \pm 1,8$ & $1,6 \pm 1,3(p=0,327)$ \\
Action errors task 1 & $3,6 \pm 2,9$ & $3,4 \pm 2,7(p=0,729)$ \\
Action errors task 2 & $0,4 \pm 0,8$ & $0,6 \pm 1,0(p=0,353)$ \\
Action errors task 3 & &
\end{tabular}

untreated migraine performed significantly worse in TMT part $B(p=0.004)$ and had a greater number of omission errors in task 1 ( $p=0.019)$ of the Visual Attention Test when compared to the migraine prophylaxis group (Table 2). The migraine prophylaxis and control group showed similar results. The only variable that was significantly different between these two groups was the reaction time in task 1 of the Visual Attention Test ( $p=$ 0.038) (Table 3).

\section{DISCUSSION}

Children with untreated migraine performed significantly worse in all tests applied in comparison to control children or children undergoing migraine prophylaxis. Nonetheless, the performance in attention tasks was within the normal range in all groups. Compared to the other groups, the untreated migraine group presented deficits in selective and alternate attention.

In this study, we were unable to evaluate differences between prophylactic treatments due to small number of children taking each type of medication. However, clinical observation revealed no performance differences between children taking different prophylactic drugs. Importantly, all the children who underwent migraine prophylaxis experienced an improvement in their symptoms, regardless of the drug being administered.

Attention is the capacity to respond to significant stimuli, irrespective of other stimuli. This is an important 
cognitive function, highly dependent on a set of anatomical structures such as the brain stem, the cerebral cortex, and the limbic system, as well as on a combination of neurotransmitters, chiefly noradrenaline and dopamine. ${ }^{(5)}$

Disturbances of these neurotransmitters can cause attention deficits. In addition, they play a role in the physiopathology of migraine, including pain and other symptoms in the crisis and intercrisis periods. ${ }^{(6-8)}$

With an effective prophylactic treatment, it is possible that the equilibrium of neurotransmitters is re-established, consequently restoring attention. This hypothesis being proposed, could attention deficit be considered a migraine symptom in some patients? Further large-scale studies are needed to address this question. It is necessary to investigate cognitive dysfunction in children with migraine, and to administer effective prophylactic treatment when indicated.

\section{REFERENCES}

1. Arruda MA, Guidetti V, Galli F, et al. Primary headaches in childhood-a population-based study. Cephalalgia. 2010; 30(9): 1056-64.

2. Riva D, Usilla A, Aggio F, et al. Attention in children and adolescents with headache. Headache. 2012;52(3): 374-84.

3. Villa TR, Correa Moutran AR, Sobirai Diaz LA, et al. Visual attention in children with migraine: a controlled comparative study. Cephalalgia. 2009; 29(6): 631-4

4. Headache Classification Committee of the International Headache Society. The International Classification of Headache Disorders: 2nd edition. Cephalalgia. 2004; 24 Suppl 1: 9-160.

5. Posner MI, and Petersen ES. The attention system of the human brain. Annual Reviews Neurosci. 1990; 13: 25-42.

6. Mulder EJ, Linssen WH, Passchier J, et al. Interictal and postictal cognitive changes in migraine. Cephalalgia. 1999;19:557-65.

7. Peroutka SJ. Migraine: a chronic sympathetic nervous system disorder. Headache 2004 ;44: 53-64.

8. Akerman S and Goadsby PJ. Dopamine and migraine: biology and clinical implications. Cephalalgia. 2007; 27:1308-14. 